\title{
A 1 Adenosine Receptor Partial Agonists and Allosteric Modulators: Advancing Toward the Clinic?
}

\author{
Fabrizio Vincenzi ${ }^{1 *}$, Silvia Pasquini ${ }^{1}$, Enrica Battistello ${ }^{1}$, Stefania Merighi ${ }^{1}$, Stefania Gessi ${ }^{1}$, \\ Pier Andrea Borea ${ }^{2}$ and Katia Varani ${ }^{1}$ \\ ${ }^{1}$ Department of Translational Medicine and for Romagna, University of Ferrara, Ferrara, Italy, ${ }^{2}$ University of Ferrara, Ferrara, Italy
}

Keywords: adenosine, positive allosteric modulators, partial agonists, $\mathbf{A}_{1}$ adenosine receptors, clinical trials

\section{INTRODUCTION}

Adenosine is an endogenous autacoid that exerts a variety of physiological effects by interacting with cell surface G-protein-coupled receptor subtypes, namely $A_{1}, A_{2 A}, A_{2 B}$, and $A_{3}$ adenosine receptors (ARs) (Borea et al., 2018). Numerous experimental evidence suggests that $A_{1} A R$ represents a promising therapeutic drug target for different pathological conditions of the central nervous system, cardiovascular disorders, and metabolic diseases (Burnstock, 2018). Through activation of pre- and post-synaptic $\mathrm{A}_{1} \mathrm{ARs}$, adenosine plays an important role as an endogenous neuroprotective modulator mainly by inhibiting the excessive release of excitatory neurotransmitters like glutamate, an effect that also underlies the anticonvulsant action of adenosine. The $\mathrm{A}_{1} \mathrm{AR}$ agonists have been recognized as promising antinociceptive agents in several preclinical models of chronic and neuropathic pain (Borea et al., 2016). The adenosinergic system also regulates mechanisms related to psychiatric disorders, where $\mathrm{A}_{1}$ ARs mediates antidepressant and anxiolyticlike effects. In the heart, adenosine acts as a cytoprotective modulator in response to stress, mostly via $\mathrm{A}_{1}$ ARs. Activation of myocardial $\mathrm{A}_{1} \mathrm{ARs}$ has been shown to exert beneficial effects against ischemic cardiac injury, arrhythmogenesis, coronary and ventricular dysfunction, and ventricular remodeling (Dinh et al., 2017). Given the role of $A_{1} A R$ stimulation to inhibit lipolysis, improve insulin sensitivity and inhibit insulin release, $A_{1} A R$ agonists have the potential to be useful for metabolic disorders such as type 2 diabetes, obesity, and hyperlipidemia (Dhalla et al., 2009a). From these premises, pharmacological agents that activate $\mathrm{A}_{1} \mathrm{ARs}$ should have huge potential for therapeutic use in different pathological conditions. Toward this goal, various selective agonists of the $\mathrm{A}_{1} \mathrm{ARs}$ are available, and some have already entered early-phase clinical trials. Nonetheless, the transition of $\mathrm{A}_{1} \mathrm{AR}$ full agonists into the clinic as effective drugs has been severely hampered due to low efficacy, receptor desensitization, and safety issues related to off-target effects. Common side effects include bradycardia, atrioventricular blocks, vasoconstriction, sedation, and antidiuretic effects. As an alternative strategy, $\mathrm{A}_{1} \mathrm{AR}$ partial agonists and positive allosteric modulators have been found to be equally effective while avoiding severe adverse effects and receptor downregulation/ desensitization. Pharmacologic and preclinical data have revealed that, as compared to full

Received: 02 November 2020 Accepted: 17 November 2020 Published: 09 December 2020

Citation:

Vincenzi $F$, Pasquini S, Battistello E, Merighi S, Gessi S, Borea PA and

Varani K (2020) $A_{1}$ Adenosine

Receptor Partial Agonists and Allosteric Modulators: Advancing

Toward the Clinic?

Front. Pharmacol. 11:625134. doi: 10.3389/fphar.2020.625134

Abbreviations: $2^{\prime}$ dCPA, 2'deoxy-N6-cyclopentyladenosine; 3'dCCPA, 3'deoxy-N6-cyclopentyladenosine; $5^{\prime}$ dCPA, 5'deoxyN6-cyclopentyladenosine; ARs, Adenosine receptors; CCPA, 2-chloro N(6)-cyclopentyladenosine; CVT-3619 (or GS-9667) [2\{6-[((1R,2R)-2-hydroxycyclopentyl)-amino]purin-9-yl\}(4S,5S,2R,3R)-5-[(2-fluorophenylthio)methyl]-oxolane-3,4-diol]; eEPSC, evoked excitatory postsynaptic currents; MCPA, 8-methylamino-N6-cyclopentyladenosine; PD 81,723 (2-Amino-4,5-dimethyl-3thienyl)-[3-(trifluoromethyl)phenyl]methanone; T62 (2-Amino-3-(4-chlorobenzoyl)-5,6,7,8-tetrahydrobenzothiophene; TRR469 (2-Amino-4-[(4-(phenyl)piperazin-1-yl)methyl]-5-(4-fluorophenyl)thiophen-3-yl)-(4-chlorophenyl)methanone; VCP171 (2amino-4-[3-(trifluoromethyl)phenyl]thiophen-3-yl)-phenylmethanone; VCP333, tert-butyl 2-amino-3-(4-chlorobenzoyl)-7,8dihydro-4H-thieno[2,3-d] azepine-6(5H)-carboxylate. 
agonists, $\mathrm{A}_{1} \mathrm{AR}$ partial agonists exert minimal effects on blood pressure, heart rate, alertness, and renal function (Greene et al., 2016). Partial agonists can be used to trigger only some of the physiological effects of $\mathrm{A}_{1} \mathrm{AR}$ activation and usually display greater tissue or organ selectivity, because of the differential receptor expression level. Furthermore, in the case where the beneficial effect is maintained, partial agonism is a suitable approach to circumvent $\mathrm{A}_{1} \mathrm{AR}$ desensitization. An option perhaps even more interesting to avoid the issues associated with the use of $A_{1} A R$ full agonist, is positive allosteric modulation (Romagnoli et al., 2015). Allosteric binding regions typically display greater sequence divergence across the receptor subtypes, providing increased selectivity for allosteric than orthosteric binding ligands. Moreover, positive allosteric modulators can enhance the responsiveness of $\mathrm{A}_{1} \mathrm{ARs}$ to endogenous adenosine within the local regions of its elevated production. This is particularly prominent for the adenosinergic system, because of the rapid increase of extracellular adenosine concentration in pathological conditions such as ischemia, trauma, inflammation, pain, and seizures. Positive allosteric modulators can therefore selectively "tune" tissue responses in the function of specific adenosine levels. Orthosteric agonists, which continuously and indiscriminately stimulate the receptors, cannot achieve this type of spatial and temporal specificity of action typical of allosteric modulators. Given their numerous advantages over full agonists, the development of partial agonists and positive allosteric modulators offer therefore the possibility to exploit the great therapeutic potential of $A_{1} A R$ activation, facilitating the transition from basic research to clinical practice. This opinion article discusses the most interesting results obtained in preclinical and clinical studies using $A_{1} A R$ partial agonists and positive allosteric modulators.

\section{PAIN AND CENTRAL NERVOUS SYSTEM DISORDERS}

One of the few studies describing the antinociceptive effect of $A_{1} A R$ partial agonists evaluated MCPA and $2^{\prime} \mathrm{dCPA}$ in the chronic constriction injury model of neuropathic pain. Despite the two partial agonists showed an anti-hyperalgesic effect comparable to that of the full agonist $5^{\prime} \mathrm{dCPA}$, their anti-nociceptive effect was lower due to the poor distribution to the site of action (Schaddelee et al., 2005). A greater number of investigations on pain have instead been performed employing $A_{1} A R$ positive allosteric modulators. The first $A_{1} A R$ positive allosteric modulators were characterized by Bruns and Fergus in 1990, the most selective of which was PD 81,723 (Bruns and Fergus, 1990). A few years later, T62, a derivative of PD 81,723 , was tested on mechanical hypersensitivity after spinal nerve ligation (Pan et al., 2001). The reduction of hyperalgesia by intrathecal injection of T62, consistent with ongoing spinal adenosine release in this model of neuropathic pain, represents the first proof of concept of the potential antinociceptive effect of $\mathrm{A}_{1} \mathrm{AR}$ positive allosteric modulators. In a subsequent study, $\mathrm{Li}$ and colleagues hypothesized the involvement of a spinal noradrenergic activation in the anti-allodynic effect of T62 (Li et al., 2002). The antinociceptive effect of T62 was then demonstrated in carrageenan- induced inflammatory ( $\mathrm{Li}$ et al., 2003) and incision-induced postoperative pain (Obata et al., 2004). Afterward, we characterized a new series of $\mathrm{A}_{1} \mathrm{AR}$ positive allosteric modulators, among which the thiophene $\mathrm{C}-5$ aryl derivative TRR 469 was selected for further studies (Romagnoli et al., 2012). TRR469 was revealed to be a more potent allosteric modulator of $\mathrm{A}_{1} \mathrm{ARs}$ compared to T62 or $\mathrm{PD} 81,723$, being able to increase by 33 fold the affinity of adenosine and showing an allosteric cooperativity factor $(\alpha)$ of 26.3 compared to 2.5 for T62 and 1.9 for PD 81,723 (Vincenzi et al., 2014). When tested for its antinociceptive action, TRR469 demonstrated to be efficacious in the formalin and writhing tests as well in the streptozotocin-induced diabetic neuropathic pain model. Furthermore, TRR469 did not display locomotor or cataleptic side effects, as opposed to the direct activating $\mathrm{A}_{1} \mathrm{AR}$ full agonist CCPA (Vincenzi et al., 2014). In the same years, Imlach and colleagues evaluated the actions of the novel $\mathrm{A}_{1} \mathrm{AR}$ positive allosteric modulator VCP171 on excitatory and inhibitory neurotransmission at spinal cord superficial dorsal horn synapses in a rat partial nerve-injury model of neuropathic pain. Electrophysiological studies showed that VCP171 inhibits eEPSC amplitude of nerve-injury animals in both lamina I and lamina II neurons (Imlach et al., 2015). These results, together with the enhanced adenosine tone at excitatory synapses in the dorsal horn after nerve injury, suggest that $A_{1} A R$ positive allosteric modulators can be effective treatments for neuropathic pain.

In addition to its antinociceptive effects, the $\mathrm{A}_{1} \mathrm{ARs}$ positive allosteric modulator TRR469 was also investigated as an anxiolytic agent. In mice, the administration of TRR469 resulted in robust anxiolytic-like effects in the elevated plus maze, the dark/light box, the open field, and the marble burying tests. Interestingly, the magnitude of the anxiolytic action of the novel $A_{1} A R$ positive allosteric modulator was comparable to that obtained with benzodiazepine diazepam (Vincenzi et al., 2016). However, in contrast to diazepam, TRR469 did not potentiate the sedative effect and locomotor disturbances elicited by ethanol.

Some studies suggested the use of partial agonists and positive allosteric modulators of $\mathrm{A}_{1} \mathrm{ARs}$ as an alternative strategy to exploit the therapeutic potential of $\mathrm{A}_{1} \mathrm{AR}$ activation against cerebral ischemic injury. $2^{\prime} \mathrm{dCCPA}$ and $3^{\prime} \mathrm{dCCPA}$, two $\mathrm{A}_{1} \mathrm{AR}$ partial agonists, protected SH-SY5Y from oxygen-glucose deprivationinduced cell viability reduction. In the same study, both compounds elicited a significant recovery of synaptic transmission in mice hippocampal slices following the application of oxygen-glucose deprivation (Martire et al., 2019). Regarding positive allosteric modulators, the effect of $\mathrm{PD} 81,723$ was evaluated on hippocampal injury and Morris water maze performance following hyperglycemic cerebral ischemia and reperfusion. Despite a high dose did not exert any effects, a lower dose of PD 81,723 resulted in a significant reduction of hippocampal injury and improvement of Morris water maze performance. The authors suggested that the presence of the allosteric modulator enhanced the neuroprotective action of the endogenously produced adenosine during hyperglycemic ischemia (Meno et al., 2003). More recently, we elucidate the role of adenosine in glutamate-induced injury in PC12 cells demonstrating that its endogenous presence is a necessary condition for the onset of cytotoxicity. This effect was attributed to the activation of Gs 
protein-coupled $A_{2 A}$ and $A_{2 B}$ ARs and counteracted by $A_{1} A R s$ stimulation. Thank to its capability to increase adenosine affinity for $\mathrm{A}_{1} \mathrm{AR}$ subtypes, the positive allosteric modulator TRR469, as opposed to CCPA, completely abrogated glutamate-mediated cell injury (Vincenzi et al., 2020).

\section{CARDIOVASCULAR SYSTEM DISEASES}

Full agonists of the $A_{1} A R s$ reduce heart rate, atrioventricular conduction, and at high doses can cause atrioventricular block. In contrast with full agonists, partial $\mathrm{A}_{1} \mathrm{AR}$ agonists are less likely to cause undesirable side effects and emerged as promising candidates for heart failure (Albrecht-Küpper et al., 2012; Greene et al., 2016). The rationale behind the beneficial effect of $A_{1} A R$ activation is mainly related to the preservation of mitochondrial function of cardiomyocytes in ischemia/reperfusion injury (Dinh et al., 2017). Two of the most studied $A_{1}$ AR partial agonists for the treatment of heart diseases are capadenoson and its derivative neladenoson bialanate. Capadenoson has undergone two phase IIa clinical trials, initially in patients with atrial fibrillation and subsequently in patients with stable angina where it lowered exercise heart rate at comparable maximum workload and improved total exercise time and prolongation of time to ischemia (Tendera et al., 2012). In an animal model of ischemic heart failure, capadenoson improved left ventricular functions and prevented maladaptive ventricular remodeling without causing atrioventricular block (Sabbah et al., 2013). Starting from capadenoson, Meibom and colleagues evaluated identified neladenoson bialanate, a prodrug of an $\mathrm{A}_{1} \mathrm{AR}$ agonist tailored to a specific partiality range, thereby optimizing the therapeutic window (Meibom et al., 2017). In two early pilot studies, the treatment of heart failure patients with reduced ejection fraction with neladenoson bialanate appeared to be safe without atrioventricular conduction disorders or neurological side effects (Voors et al., 2017). Recently, two phase IIb clinical trials were conducted to assess the dose-response effect of neladenoson bialanate on cardiac structure and function, clinical outcome, and safety in patients with heart failure with reduced ejection fraction (Voors et al., 2019) or preserved ejection fraction (Bertero and Maack, 2019). Unfortunately, no dose-dependent changes in the primary or secondary endpoints were observed in patients randomized to neladenoson compared with placebo. Among patients with heart failure with preserved ejection fraction, there was no significant dose-response relationship detected for neladenoson concerning the change in exercise capacity from baseline to 20 weeks (Shah et al., 2019). The lack of effect of these partial agonists underlines the need to find new ways to exploit the potential of $A_{1}$ ARs. A recent and interesting structure modification in capadenoson derivatives yielded the covalent partial agonist LUF774, which may serve as a prototype for a novel therapeutic approach (Yang et al., 2020). As for positive allosteric modulators, no clinical trials have been conducted for heart diseases to date. However, in one of the few preclinical studies, the positive allosteric modulator of the $\mathrm{A}_{1} \mathrm{ARs} \mathrm{VCP} 333$ improved cardiac function and reduced myocardial cell death following ischemia-reperfusion injury in murine isolated heart (Butcher et al., 2013).

\section{METABOLIC DISEASES}

The evidence from numerous studies indicates that $A_{1} A R s$ are potent inhibitors of adipose tissue lipolysis. From a clinical point of view, the availability of pharmacological agents that inhibit lipolysis and lower circulating free fatty acid levels may be useful in the treatment of type 2 diabetes. In this context, various preclinical studies have been carried out employing CVT-3619, an $A_{1}$ AR partial agonist. In rats, CVT-3619 significantly reduced plasma free fatty acid levels at doses that did not elicit cardiovascular side effects (Dhalla et al., 2007a). In subsequent studies, CVT-3619 treatment lowered circulating free fatty acid and triglyceride concentrations and improves insulin sensitivity and glucose clearance in animals with dietary-induced forms of insulin resistance (Dhalla et al., 2007b; Shearer et al., 2009) and in diabetic rats (Dhalla et al., 2009b). Under the name of GS-9667, CVT-3619 was tested in two clinical studies to evaluate its efficacy and safety. In healthy non-obese and obese subjects, the $A_{1} A R$ partial agonist GS-9667 reduced plasma free fatty acid levels, exhibited linear kinetics, and was well-tolerated, representing therefore a promising therapy for type 2 diabetes and dyslipidemia (Staehr et al., 2013).

\section{CONCLUSION}

Activation of $\mathrm{A}_{1} \mathrm{ARs}$ has huge untapped therapeutic potential. Unfortunately, their ubiquitous distribution together with receptor desensitization and off-target effect of $A_{1} A R$ full agonists has greatly hindered their exploitation in the clinic. Different pharmacological approaches such as partial agonists and positive allosteric modulators could offer significant advantages over $\mathrm{A}_{1} \mathrm{AR}$ full agonists. Despite the promising preclinical evidence, no clinical trials have so far been performed on $\mathrm{A}_{1} \mathrm{AR}$ positive allosteric modulators. We think that these compounds, especially the most potent ones like TRR469 or VCP171, could represent in the future a possible way to improving translation from promising preclinical results to clinical success.

\section{AUTHOR CONTRIBUTIONS}

$\mathrm{FV}$, SP, $\mathrm{PB}$, and $\mathrm{KV}$ conceived the work and wrote the manuscript. EB, SM, and SG contributed to writing and editing. All authors contributed to the article and approved the submitted version.

\section{ACKNOWLEDGMENTS}

We dedicate this opinion article to the memory of Geoffrey Burnstock, a pioneer scientist whose groundbreaking discoveries laid the foundation in the field of purinergic signaling and represents a source of encouragement and inspiration for all the pharmacologists who wants to contribute to this exciting field of research. 


\section{REFERENCES}

Albrecht-Küpper, B. E., Leineweber, K., and Nell, P. G. (2012). Partial adenosine A1 receptor agonists for cardiovascular therapies. Purinergic Signal. 8, 91-99. doi:10.1007/s11302-011-9274-3

Bertero, E., and Maack, C. (2019). The partial AdeNosine Al receptor agonist in patients with chronic heart failure and preserved ejection fraction (PANACHE) trial. Cardiovasc. Res. 115, e71-e73. doi:10.1093/cvr/cvz123

Borea, P. A., Gessi, S., Merighi, S., and Varani, K. (2016). Adenosine as a multisignalling guardian angel in human diseases: when, where and how does it exert its protective effects? Trends Pharmacol. Sci. 37, 419-434. doi:10.1016/j.tips. 2016.02.006

Borea, P. A., Gessi, S., Merighi, S., Vincenzi, F., and Varani, K. (2018). Pharmacology of adenosine receptors: the state of the art. Physiol. Rev. 98, 1591-1625. doi:10.1152/physrev.00049.2017

Bruns, R. F., and Fergus, J. H. (1990). Allosteric enhancement of adenosine A1 receptor binding and function by 2-amino-3-benzoylthiophenes. Mol. Pharmacol. 38, 939-949.

Burnstock, G. (2018). The therapeutic potential of purinergic signalling. Biochem. Pharmacol. 151, 157-165. doi:10.1016/j.bcp.2017.07.016

Butcher, A., Scammells, P. J., White, P. J., Devine, S. M., and Rose'meyer, R. B. (2013). An allosteric modulator of the adenosine Al receptor improves cardiac function following ischaemia in murine isolated hearts. Pharm. Basel Switz. 6, 546-556. ddoi:10.3390/ph6040546

Dhalla, A. K., Santikul, M., Smith, M., Wong, M.-Y., Shryock, J. C., and Belardinelli, L. (2007a). Antilipolytic activity of a novel partial A1 adenosine receptor agonist devoid of cardiovascular effects: comparison with nicotinic acid. J. Pharmacol. Exp. Therapeut. 321, 327-333. doi:10. 1124/jpet.106.114421

Dhalla, A. K., Wong, M. Y., Voshol, P. J., Belardinelli, L., and Reaven, G. M. (2007b). A1 adenosine receptor partial agonist lowers plasma FFA and improves insulin resistance induced by high-fat diet in rodents. Am. J. Physiol. Endocrinol. Metab. 292, E1358-E1363. doi:10.1152/ajpendo.00573. 2006

Dhalla, A. K., Chisholm, J. W., Reaven, G. M., and Belardinelli, L. (2009a). A1 adenosine receptor: role in diabetes and obesity. Handb. Exp. Pharmacol., 271-295. doi:10.1007/978-3-540-89615-9_9

Dhalla, A. K., Santikul, M., Chisholm, J. W., Belardinelli, L., and Reaven, G. M. (2009b). Comparison of the antilipolytic effects of an A1 adenosine receptor partial agonist in normal and diabetic rats. Diabetes Obes. Metabol. 11, 95-101. doi:10.1111/j.1463-1326.2008.00902.x

Dinh, W., Albrecht-Küpper, B., Gheorghiade, M., Voors, A. A., van der Laan, M., and Sabbah, H. N. (2017). Partial adenosine Al agonist in heart failure. Handb. Exp. Pharmacol. 243, 177-203. doi:10.1007/164_2016_83

Greene, S. J., Sabbah, H. N., Butler, J., Voors, A. A., Albrecht-Küpper, B. E., Düngen, H.-D., et al. (2016). Partial adenosine A1 receptor agonism: a potential new therapeutic strategy for heart failure. Heart Fail. Rev. 21, 95-102. doi:10. 1007/s10741-015-9522-7

Imlach, W. L., Bhola, R. F., May, L. T., Christopoulos, A., and Christie, M. J. (2015). A positive allosteric modulator of the adenosine A1 receptor selectively inhibits primary afferent synaptic transmission in a neuropathic pain model. Mol. Pharmacol. 88, 460-468. doi:10.1124/mol. 115.099499

Li, X., Conklin, D., Ma, W., Zhu, X., and Eisenach, J. C. (2002). Spinal noradrenergic activation mediates allodynia reduction from an allosteric adenosine modulator in a rat model of neuropathic pain. Pain 97, 117-125. doi:10.1016/s0304-3959(02)00011-8

Li, X., Conklin, D., Pan, H.-L., and Eisenach, J. C. (2003). Allosteric adenosine receptor modulation reduces hypersensitivity following peripheral inflammation by a central mechanism. J. Pharmacol. Exp. Therapeut. 305, 950-955. doi:10.1124/jpet.102.047951

Martire, A., Lambertucci, C., Pepponi, R., Ferrante, A., Benati, N., Buccioni, M., et al. (2019). Neuroprotective potential of adenosine Al receptor partial agonists in experimental models of cerebral ischemia. J. Neurochem. 149, 211-230. doi:10.1111/jnc. 14660

Meibom, D., Albrecht-Küpper, B., Diedrichs, N., Hübsch, W., Kast, R., Krämer, T., et al. (2017). Neladenoson bialanate hydrochloride: a prodrug of a partial adenosine A1 receptor agonist for the chronic treatment of heart diseases. ChemMedChem 12, 728-737. doi:10.1002/ cmdc. 201700151

Meno, J. R., Higashi, H., Cambray, A. J., Zhou, J., D’Ambrosio, R., and Winn, H. R. (2003). Hippocampal injury and neurobehavioral deficits are improved by PD 81,723 following hyperglycemic cerebral ischemia. Exp. Neurol. 183, 188-196. doi:10.1016/s0014-4886(03)00162-6

Obata, H., Li, X., and Eisenach, J. C. (2004). Spinal adenosine receptor activation reduces hypersensitivity after surgery by a different mechanism than after nerve injury. Anesthesiology 100, 1258-1262. doi:10.1097/00000542-20040500000030

Pan, H. L., Xu, Z., Leung, E., and Eisenach, J. C. (2001). Allosteric adenosine modulation to reduce allodynia. Anesthesiology 95, 416-420. doi:10.1097/ 00000542-200108000-00025

Romagnoli, R., Baraldi, P. G., Carrion, M. D., Cara, C. L., Cruz-Lopez, O., Salvador, M. K., et al. (2012). Synthesis and biological evaluation of 2-amino-3-(4chlorobenzoyl)-4-[(4-arylpiperazin-1-yl)methyl]-5-substituted-thiophenes. effect of the 5-modification on allosteric enhancer activity at the $\mathrm{Al}$ adenosine receptor. J. Med. Chem. 55, 7719-7735. doi:10.1021/jm3007504

Romagnoli, R., Baraldi, P. G., Moorman, A. R., Borea, P. A., and Varani, K. (2015) Current status of Al adenosine receptor allosteric enhancers. Future Med. Chem. 7, 1247-1259. doi:10.4155/fmc. 15.65

Sabbah, H. N., Gupta, R. C., Kohli, S., Wang, M., Rastogi, S., Zhang, K., et al. (2013). Chronic therapy with a partial adenosine Al-receptor agonist improves left ventricular function and remodeling in dogs with advanced heart failure. Circ. Heart Fail 6, 563-571. doi:10.1161/ CIRCHEARTFAILURE.112.000208

Schaddelee, M. P., Collins, S. D., DeJongh, J., de Boer, A. G., Ijzerman, A. P., and Danhof, M. (2005). Pharmacokinetic/pharmacodynamic modelling of the antihyperalgesic and anti-nociceptive effect of adenosine A1 receptor partial agonists in neuropathic pain. Eur. J. Pharmacol. 514, 131-140. doi:10.1016/j. ejphar.2005.03.026

Shah, S. J., Voors, A. A., McMurray, J. J. V., Kitzman, D. W., Viethen, T., Bomfim Wirtz, A., et al. (2019). Effect of neladenoson bialanate on exercise capacity among patients with heart failure with preserved ejection fraction: a randomized clinical trial. J.AMA 321, 2101-2112. doi:10.1001/jama.2019. 6717

Shearer, J., Severson, D. L., Su, L., Belardinelli, L., and Dhalla, A. K. (2009). Partial A1 adenosine receptor agonist regulates cardiac substrate utilization in insulinresistant rats in vivo. J. Pharmacol. Exp. Therapeut. 328, 306-311. doi:10.1124/ jpet.108.143594

Staehr, P. M., Dhalla, A. K., Zack, J., Wang, X., Ho, Y. L., Bingham, J., et al. (2013). Reduction of free fatty acids, safety, and pharmacokinetics of oral GS-9667, an A(1) adenosine receptor partial agonist. J. Clin. Pharmacol. 53, 385-392. doi:10. 1002/jcph.9

Tendera, M., Gaszewska-Żurek, E., Parma, Z., Ponikowski, P., Jankowska, E., Kawecka-Jaszcz, K., et al. (2012). The new oral adenosine A1 receptor agonist capadenoson in male patients with stable angina. Clin. Res. Cardiol. 101, 585-591. doi:10.1007/s00392-012-0430-8

Vincenzi, F., Targa, M., Romagnoli, R., Merighi, S., Gessi, S., Baraldi, P. G., et al. (2014). TRR469, a potent $\mathrm{A}(1)$ adenosine receptor allosteric modulator, exhibits anti-nociceptive properties in acute and neuropathic pain models in mice. Neuropharmacology 81, 6-14. doi:10.1016/j.neuropharm.2014.01. 028

Vincenzi, F., Pasquini, S., Gessi, S., Merighi, S., Romagnoli, R., Borea, P. A., et al. (2020). The detrimental action of adenosine on glutamate-induced cytotoxicity in PC12 cells can be shifted towards a neuroprotective role through A1AR positive allosteric modulation. Cells 9, 1242. doi:10.3390/ cells 9051242

Vincenzi, F., Ravani, A., Pasquini, S., Merighi, S., Gessi, S., Romagnoli, R., et al. (2016). Positive allosteric modulation of A1 adenosine receptors as a novel and promising therapeutic strategy for anxiety. Neuropharmacology 111, 283-292. doi:10.1016/j.neuropharm.2016.09. 015

Voors, A. A., Bax, J. J., Hernandez, A. F., Wirtz, A. B., Pap, A. F., Ferreira, A. C., et al. (2019). Safety and efficacy of the partial adenosine Al receptor agonist neladenoson bialanate in patients with chronic heart failure with reduced ejection fraction: a phase IIb, randomized, double-blind, 
placebo-controlled trial. Eur. J. Heart Fail. 21, 1426-1433. doi:10.1002/ ejhf.1591

Voors, A. A., Düngen, H.-D., Senni, M., Nodari, S., Agostoni, P., Ponikowski, P., et al. (2017). Safety and tolerability of neladenoson bialanate, a novel oral partial adenosine A1 receptor agonist, in patients with chronic heart failure. J. Clin. Pharmacol. 57, 440-451. doi:10.1002/jcph. 828

Yang, X., Dilweg, M. A., Osemwengie, D., Burggraaff, L., van der Es, D., Heitman, L. H., et al. (2020). Design and pharmacological profile of a novel covalent partial agonist for the adenosine A1 receptor. Biochem. Pharmacol. 180, 114144. doi:10.1016/j.bcp.2020.114144
Conflict of Interest: The authors declare that the research was conducted in the absence of any commercial or financial relationships that could be construed as a potential conflict of interest.

Copyright $\odot 2020$ Vincenzi, Pasquini, Battistello, Merighi, Gessi, Borea and Varani. This is an open-access article distributed under the terms of the Creative Commons Attribution License (CC BY). The use, distribution or reproduction in other forums is permitted, provided the original author(s) and the copyright owner(s) are credited and that the original publication in this journal is cited, in accordance with accepted academic practice. No use, distribution or reproduction is permitted which does not comply with these terms. 